\title{
LOS PROBLEMAS DE LÁPIZ Y PAPEL EN LA FORMACIÓN DE PROFESORES
}

\author{
MARTÍNEZ LOSADA, CRISTINA, GARCÍA BARROS, SUSANA, MONDELO ALONSO, MATILDE \\ y VEGA MARCOTE, PEDRO \\ Facultade de Ciencias da Educación. Universidade da Coruña. Paseo de Ronda, 47. 15011 A Coruña.
}

\begin{abstract}
SUMMARY
In this article a didactic approach for both initial and ongoing teacher training is presented, oriented towards the teaching and learning of paper and pencil problem-solving. Taking as a starting point teachers' ideas of the educational possibilities of the problems which are generally used in Science classes (objectives reached and specific procedures which they develop), it then goes on to analyse and evaluate concrete traditional and innovative problem-solving models. This study will also look at commentaries and results regarding the development of these activities from both in-service and trainee teachers. It is shown that these teachers initially accept the educational interest of such problems, and they consider the innovative model to be the most useful because, apart from motivating the students, it also develops research procedures which the traditional problems ignore, and which are fundamental in scientific training.
\end{abstract}

\section{INTRODUCCIÓN}

Los problemas de lápiz y papel son una actividad habitual en la clase de ciencias cuyo valor formativo es ampliamente reconocido por el profesorado (Garrett, 1986). La resolución de problemas afianza y promueve el conocimiento de los estudiantes porque permite aplicar los aspectos teóricos a situaciones prácticas específicas (Pozo et al., 1995; Selvaratnman, 1983). Por otra parte los problemas, considerados en sentido amplio, favorecen el desarrollo y el aprendizaje de procedimientos, destrezas y actitudes científicas contempladas en los currículos de ciencias de la enseñanza obligatoria de nuestro país. Otra aportación importante de estas actividades es que ayudan al estudiante a «aprender a aprender», permitiendo aplicar sus conocimientos a la resolución de problemas de la vida diaria (Pozo et al., 1994) y a desarrollar el pensamiento creativo (Garrett, 1988).

Existen distintos tipos de problemas que pueden ser clasificados en función de diferentes criterios. Perales
(1993) establece tres categorías: $a$ ) según el campo de conocimiento aplicado distingue los cotidianos y los académicos; $b$ ) según el tipo de tarea agrupa los cuantitativos, que demandan determinaciones numéricas, y los cualitativos, más centrados en la interpretación científica de fenómenos reales, denominados habitualmente cuestiones; $c$ ) en cuanto a la naturaleza del enunciado y a las características del proceso de resolución diferencia los problemas cerrados, fácilmente resolubles mediante la utilización de determinados algoritmos, de los abiertos, que demandan la utilización del pensamiento productivo para el diseño de estrategias de resolución. Con relación a este último aspecto, Garrett (1988) distingue entre ejercicios-puzzles y problemas verdaderos, en función de los intereses y conocimientos del resolvente. Los primeros implican reconocimiento y reproducción, mientras que los segundos suponen un proceso de construcción (Dumas-Carré y Larchen, 1987). De todo esto se deduce que pueden plantearse problemas con fines educativos 
diferentes. Así, siguiendo a Caballer y Oñorbe (1997), cabría distinguir entre «problemas-cuestiones», para reforzar y aplicar la teoría; «problemas-ejercicio», para el aprendizaje de técnicas de resolución ya establecidas que permitan alcanzar una solución correcta; y «problemasinvestigación», entre los que se incluirían tambien actividades de laboratorio, útiles para el desarrollo de procedimientos y actitudes hacia la ciencia y sus métodos de trabajo. En esta línea, Watts (1991) distingue dos tipos de problemas: $a$ ) los PS1 (problem-solving 1) que incluyen aquéllos más tradicionales, asociados a los problemas académicos de las clases de ciencias, cuyos enunciados están bien definidos y cuya resolución se basa en procesos puramente intelectuales; $b$ ) los problemas PS2, más genéricos, generalmente cualitativos y próximos a la vida real, que pueden requerir práctica experimental y cuyo objetivo fundamental es el desarrollo de estrategias de resolución, implicando a los ámbitos cognitivo, manipulativo y afectivo.

La investigación en resolución de problemas ha puesto de manifiesto que este tipo de actividades no resulta fácil para los alumnos. Éstos tienden a abordar los problemas escolares del mismo modo que abordan los problemas cotidianos, centrando sus esfuerzos en alcanzar el resultado correcto y olvidándose de la comprensión del proceso (Pozo et al., 1994). Por ello, tienden a resolverlos de forma mecánica y operativa, poniendo el énfasis en la búsqueda de la fórmula adecuada (Mettes et al., 1980) y llegando incluso, en ocasiones, a la solución correcta sin haber entendido lo que han hecho (Gilbert, 1980). Además, los estudiantes tienen dificultades tanto con la utilización del razonamiento lógico y con la comprensión del enunciado del problema y la representación mental del mismo (Genyea, 1983) como con los conocimientos teóricos implicados en su resolución, que generalmente son insuficientes y están mal estructurados (Garrett, 1987; Kempa, 1986). A su vez, la escasa capacidad de los alumnos para abordar nuevos problemas, diferentes a los resueltos en clase, ha sido señalada en algunas investigaciones (Gil y Martínez Torregrosa, 1983; Gilbert 1980; Mettes et al., 1980).

Estas dificultades del alumnado en la resolución de problemas justifican la existencia de distintas líneas de investigación al respecto. Una de ellas se centró en el análisis de cómo los abordan los expertos y los novatos, con el fin de formular modelos útiles para su enseñanzaaprendizaje. Camacho y Good (1989) realizaron una interesante síntesis de distintos trabajos en este sentido, concluyendo que los expertos dedican tiempo suficiente al análisis del problema -lo que permite realizar la representación de la situación-; que poseen unos conocimientos bien coordinados y estructurados; que desarrollan estrategias poderosas de resolución, y que realizan una continua contrastación del trabajo que están realizando. Los novatos, en cambio, adoptan comportamientos menos reflexivos, basados fundamentalmente en técnicas de ensayo/error, y poseen, en general, conocimientos teóricos insuficientemente estructurados.

Otras investigaciones se orientaron al desarrollo de modelos de resolución de problemas, con el objetivo de mejorar su enseñanza-aprendizaje. Modelos herederos de las propuestas pioneras de Polya (1965) ofrecen un modo sistemático de resolver problemas (Mettes, 1980; Kramers-Pals et al., 1983), siguiendo las siguientes fases: $a$ ) análisis del problema; $b$ ) planteamiento de una estrategia de resolución que consiste básicamente en estandarizar el problema; $c$ ) ejecución de las operaciones pertinentes; $d$ ) análisis de resultados. Otro tipo de modelos de resolución, fundamentados en la historia y la filosofía de la ciencia y en la visión constructivista del aprendizaje, pretenden abordar los problemas de forma coherente con las características del trabajo científico, favoreciendo la creatividad, la reflexión, la utilización adecuada de la teoría, la formulación de hipótesis, la búsqueda de estrategias... (Caillot y Dumas Carré, 1987; Gil y Martínez Torregrosa, 1983; Lopes et al., 1996; Watts, 1991), promoviendo en los alumnos el desarrollo del «pensamiento productivo» y una mejora efectiva en su capacidad para abordar y resolver problemas. De este modo, la utilización de modelos innovadores en la enseñanza de las ciencias permitiría el desarrollo de importantes procedimientos científicos que no promueven los modelos de resolución tradicionales (Ramírez et al., 1994).

La investigación en resolución de problemas no ha tenido, sin embargo, una apreciable repercusión en las aulas, donde se sigue empleando una metodología tradicional de enseñanza de los mismos. Ésta se caracteriza por la utilización de problemas-ejercicio, llamados también problemas-tipo, explicados por el profesor a través de una serie de pasos estandarizados que alcanza la solución correcta. Posteriormente se plantean otros problemas similares que el alumno debe resolver y que finalmente formarán parte de la evaluación (DumasCarré, 1987). Por otra parte, los problemas contenidos en los libros de texto y en los específicos de problemas utilizados mayoritariamente por el profesorado siguen básicamente la misma línea (Garrett et al., 1990; Ramírez et al., 1994). Estos modelos de resolución están en consonancia con la idea de «desproblematizar» los problemas (Mettes, 1980), que consiste básicamente en la utilización de orientaciones algorítmicas. Tal desproblematización favorece la memorización y aplicación de conocimientos y reglas aprendidas, promoviendo el desarrollo de un reducido número de procedimientos científicos. Así, este tipo de problemas permite alcanzar unos objetivos muy concretos, siendo claramente insuficientes para conseguir el desarrollo de los distintos tipos de contenidos propuestos actualmente en los currículos de ciencias. Es necesario, pues, ampliar el ámbito de los problemas, tanto en cuanto al contenido como a su naturaleza y a los requerimientos necesarios para su resolución. Pero además es imprescindible, dado el alto índice de fracasos en la resolución de problemas propuestos en los exámenes, que se produzca un replanteamiento de las formas tradicionales de enseñanza hacia otras más efectivas (Caballer y Oñorbe, 1997), lo que demanda la paralela formación del profesorado. Ésta deberá basarse en la visión constructivista del aprendizaje y tomar como punto de referencia las ideas de los docentes (Briscoe, 1991), coherentes generalmente con enfoques metodológicos tradicionales. Concretamente 
con respecto a la enseñanza-aprendizaje de los problemas de lápiz y papel, una de las ideas clave del pensamiento espontáneo del profesorado es considerar que las dificultades de los alumnos en la resolución de problemas y su alto índice de fracaso radican fundamentalmente en los propios estudiantes. Su falta de conocimiento teórico, el escaso dominio del cálculo matemático, la incomprensión del enunciado... son causas de tal fracaso (Gil y Martínez-Torregrosa, 1984), mientras que la influencia del proceso de enseñanza es, a menudo, desestimada (Oñorbe y Sánchez, 1996). Por ello, la formación docente ha de favorecer el imprescindible «cambio didáctico» en el profesorado -en ejercicio o en formacióna partir del cuestionamiento de sus propias ideas (Furió, 1994; Hewson y Hewson, 1987). En este proceso juega un papel fundamental el análisis y las reflexiones críticas tanto de los planteamientos habituales, con el fin de que los profesores perciban sus deficiencias, como de otros alternativos innovadores realmente viables (Gil y Pessoa, 1994). Ello posibilitará que el docente opte por diferentes tipos de problemas en función de los objetivos que se pretendan alcanzar y supere la exclusiva utilización de modelos de resolución algorítmicos que restringen, como ya señalamos, el desarrollo de contenidos científicos.

De acuerdo con lo anteriormente indicado, en este trabajo planteamos una actividad concreta para la formación docente, desarrollada con profesores en ejercicio y en formación. El objetivo de la misma es promover la reflexión de los docentes sobre las posibilidades educativas de los problemas de lápiz y papel y el uso del análisis crítico para la valoración de diferentes problemas y de sus modelos de resolución. Además presentamos los resultados obtenidos respecto a las ideas iniciales de los profesores sobre resolución de problemas y la valoración que ellos hacen de dos modelos concretos de resolución.

\section{METODOLOGÍA}

La actividad que sobre resolución de problemas presentamos a continuación fue desarrollada en distintos cursos de formación del profesorado de ciencias, y se integran en una unidad específicamente diseñada para estudiar actividades dirigidas a la enseñanza-aprendizaje de las ciencias. Esta unidad tenía por objeto que los profesores reflexionaran sobre el interés educativo de las mismas, analizaran las ventajas y las limitaciones de los planteamientos habitualmente utilizados en el aula y evaluaran nuevos materiales.

La investigación se realizó con un total de 173 profesores en formación y en ejercicio, agrupados en: a) grupo I, 33 alumnos de $3^{\circ}$ de magisterio que cursaban la asignatura de Didáctica de la Física y Química correspondiente al plan de estudios vigente hasta el curso 1995-96 en nuestra Universidad; b) grupo II, 77 licenciados en ciencias (biología, física o química) que asistían al Seminario de Didáctica de las Ciencias incluido en el curso de aptitud pedagógica (CAP); c) grupo III,
63 profesores de educación secundaria en ejercicio, especialistas en ciencias naturales y en física y química que estaban realizando un curso de formación permanente sobre la enseñanza de las ciencias de la naturaleza en la ESO.

El planteamiento de la actividad, elaborado por nosotros, contempla básicamente dos apartados, esquematizados en la figura 1.

En el primer apartado se ponen de manifiesto y se discuten las ideas de los profesores sobre resolución de problemas: a) Los docentes, en respuesta a una pregunta abierta, emiten sus opiniones sobre las posibilidades educativas de los problemas escolares. Seguidamente analizan, en pequeño grupo, las posibilidades educativas reales de los problemas utilizados habitualmente en el aula. Con el fín de facilitar el análisis y la explicitación de ideas se suministró una guía (Anexo I) que incluye una selección de objetivos y de procedimientos científicos concretos que se podrían desarrollar con este tipo de actividades. b) Se realiza una discusión de las ideas explicitadas por los distintos grupos de trabajo. Éstos deben exponer, además, las causas de que los problemas habituales no permitan desarrollar ciertos objetivos y procedimientos. Tales opiniones fueron recogidas por escrito directamente en el transcurso de la discusión, aunque, con objeto de completar la información así obtenida, se realizó también una recogida cuantitativa de sus opiniones en algunos grupos (concretamente con 44 alumnos del CAP y 41 profesores en ejercicio). Para ello se utilizó un cuestionario específico, inspirado en el propuesto por Oñorbe y Sánchez (1996) (Anexo II).

En el segundo apartado se suministran dos tipos de problemas resueltos, uno tradicional A y otro alternativo B y se comparan sus modelos de resolución. Los profesores deben señalar los objetivos y procedimientos que permiten desarrollar ambos problemas, utilizando para ello la guía recogida en el anexo I. No se ha incluido el análisis de procedimientos de comunicación de resultados al considerar que éstos pueden favorecerse o no en ambos tipos de problemas, dependiendo de la metodología utilizada. Seguidamente se realiza una discusión en gran grupo, elaborándose las consideraciones finales oportunas.

Con el fin de adaptar los problemas a la especialidad de los profesores (biología, física y química) se utilizaron dos ejemplificaciones conocidas y fácilmente abordables por los sujetos de los tres colectivos. En una de ellas, el problema tradicional A y su correspondiente alternativo B se referían al estudio del movimiento ascendente de un objeto. En la otra ejemplificación, ambos problemas, A y B, trataban sobre la herencia de los grupos sanguíneos (Anexo III). En cualquier caso, los profesores tenían plena libertad de elección. Los dos tipos de problemas propuestos A y $\mathrm{B}$ requieren el conocimiento y manejo de los mismos contenidos conceptuales y de las mismas técnicas operativas aunque su planteamiento es diferente. El problema A es de simple aplicación de la teoría y similar a los muchos que existen en los textos comúnmente utilizados. Se trata de un problema cerra- 
do, que especifica en su resolución los aspectos teóricos que es preciso utilizar y los pasos a seguir hasta obtener la solución. El problema B es abierto y su resolución se plantea como una actividad investigativa guiada por el profesor y fundamentada en la visión constructivista del aprendizaje (Gil y Martínez-Torregrosa, 1983). En él se propone la realización de un análisis de la situación planteada y se presentan cuestiones que instan a los alumnos a proponer hipótesis y posibles estrategias de resolución. Éstos deben llevar a cabo la estrategia, obtener unos resultados y contrastarlos. El papel del docente consiste en reorientar las ideas de los alumnos, proporcionarles el apoyo teórico-práctico necesario y fomentar el intercambio y discusión entre los distintos grupos de trabajo. En este sentido, la actividad incluye notas para el profesor. Debemos señalar además que, en el momento de su presentación, se indicó a los profesores la necesidad de integrar adecuadamente estas actividades en una unidad didáctica concreta.

Para averiguar si existen o no diferencias significativas entre las opiniones de los distintos colectivos sobre las posibilidades educativas de los problemas habituales de aula se utilizó la prueba estadística del chi-cuadrado, tanto para cada uno de los objetivos como para cada uno de los procedimientos propuestos para su análisis (Anexo I), elaborándose las correspondientes tablas de contingencia. La misma estadística se usó para comparar las opiniones de los distintos grupos de profesores sobre los objetivos y procedimientos que permiten desarrollar el problema A y el problema B. De igual forma, se han

Figura 1

Esquema de la actividad desarrollada con profesores en ejercicio y en formación.

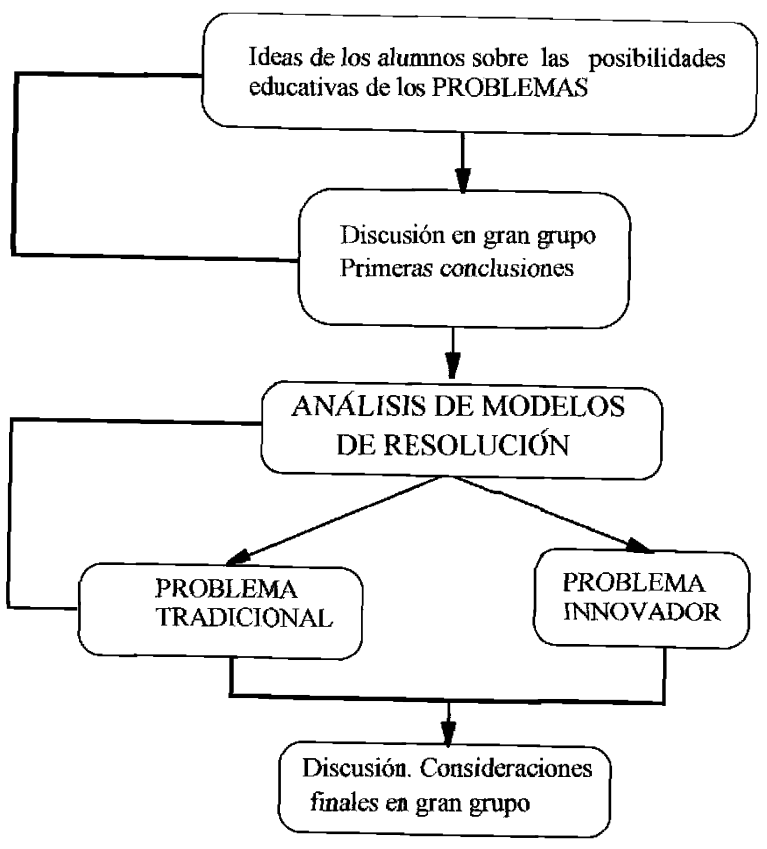

comparado las opiniones iniciales de los profesores sobre las posibilidades educativas reales de los problemas con las vertidas sobre el problema tradicional A. En todos los casos se ha tomado un nivel de significación del $0,01(\mathrm{p}=0,01)$.

\section{ANÁLISIS DE RESULTADOS}

Presentamos a continuación los resultados de la actividad realizada con profesores en ejercicio y en formación, siguiendo los dos apartados en que hemos dividido nuestro planteamiento (Fig. 1).

\section{Ideas de los profesores sobre las posibilidades educativas de los problemas de lápiz y papel}

Los profesores, en respuesta a la pregunta abierta, admitieron que los problemas son un tipo de actividad especialmente útil, que contribuye al desarrollo de los distintos tipos de contenidos que hoy se plantean en la enseñanza de las ciencias. Algunas respuestas textuales, que ilustran las opiniones expresadas mayoritariamente por los distintos colectivos son:

- Permiten aplicar la teoría a situaciones concretas; facilitan la asimilación-comprensión de conceptos.

- Se clarifican aspectos teóricos; desarrollan la capacidad de razonamiento.

- Hacen pensar al alumno.

- Promueven la adquisición y manejo de procedimientos y habilidades científicas.

- Desarrollan actitudes de orden, rigurosidad...

- Despiertan la curiosidad y el interés por resolver nuevas situaciones.

Las opiniones de los profesores en ejercicio y en formación (Anexo I) respecto a los objetivos que realmente se alcanzan con los problemas habituales de aula, se recogen en la tabla I. En ella se aprecia que una gran mayoría de los sujetos de los tres colectivos coincidió en señalar que los problemas habituales sirven para aplicar los conocimientos teóricos, previamente estudiados, a situaciones concretas. Sin embargo tal acuerdo no se traslada a los otros objetivos. Así, mientras que el 87,9\% de los alumnos de magisterio (grupo I) admitió que los problemas favorecen el aprendizaje y la comprensión de conceptos, tal porcentaje descendió significativamente con relación a los obtenidos en los grupos II y III $\left(\mathrm{X}^{2}=9,11\right.$ y 10,42 respectivamente $)$. La posibilidad de que los problemas desarrollen capacidades intelectuales y motiven a los alumnos es considerada asimismo en mayor medida en el grupo I (porcentajes superiores al $50 \%$ ) que en los otros dos grupos (entre el $35 \%$ y el $20 \%$ ). Cabe resaltar aquí la existencia de diferencias significativas entre la postura de los profesores en ejer- 
Tabla I

Opiniones de los profesores, expresadas en porcentaje, sobre los objetivos que se alcanzan con los problemas que habitualmente se utilizan.

\begin{tabular}{|l|c|c|c|}
\hline OBJETIVOS & $\begin{array}{c}\text { Grupo I } \\
(\mathbf{n = 3 3})\end{array}$ & $\begin{array}{c}\text { Grupo II } \\
(\mathbf{n}=\mathbf{7 7})\end{array}$ \\
\hline Aplicar la teoría estudiada & 100 & 87,0 & 100 \\
\hline Favorecer la comprensión & 87,9 & 55,8 & 52,4 \\
\hline Desarrollar capacidades & 63,6 & 35,1 & 22,2 \\
\hline Interesar al alumno & 51,5 & 33,8 & 20,6 \\
\hline
\end{tabular}

Tabla II

Opiniones de los profesores, expresadas en porcentaje, sobre la contribución de los problemas escolares al desarrollo de procedimientos.

\begin{tabular}{|c|c|c|c|}
\hline OBJETIVOS & $\begin{array}{l}\text { Grupo I } \\
(\mathbf{n}=33)\end{array}$ & $\begin{array}{l}\text { Grupo II } \\
(\mathbf{n}=77)\end{array}$ & $\begin{array}{c}\text { Grupo III } \\
(\mathbf{n}=63)\end{array}$ \\
\hline Ejercitar técnicas específicas & 97,0 & 96,1 & 100 \\
\hline $\begin{array}{l}\text { Analizar el problema utilizando } \\
\text { los conocimientos teóricos disponibles }\end{array}$ & 84,8 & 57,1 & 42,9 \\
\hline $\begin{array}{l}\text { Proponer posibles soluciones y diseñar } \\
\text { estrategias de resolución }\end{array}$ & 48,5 & 20,8 & 11,1 \\
\hline $\begin{array}{l}\text { Comprobar que se ha cumplido } \\
\text { la hipótesis propuesta }\end{array}$ & 45,5 & 20,8 & 19,0 \\
\hline Analizar los resultados obtenidos & 39,4 & 42,9 & 41,3 \\
\hline Contrastar opiniones con otros compañeros & 45,5 & 48,1 & 42,9 \\
\hline $\begin{array}{l}\text { Exponer por escrito el proceso seguido } \\
\text { en la resolución }\end{array}$ & 42,4 & 36,4 & 36,5 \\
\hline $\begin{array}{l}\text { Comunicar los resultados obtenidos } \\
\text { y discutirlos en gran grupo }\end{array}$ & 36,4 & 31,2 & 36,5 \\
\hline
\end{tabular}

cicio, que fue especialmente crítica respecto a estos dos objetivos, y la de los alumnos de magisterio $\left(X^{2}=14,3\right.$ y 8,23 respectivamente).

Los procedimientos específicos que se desarrollan con los problemas habitualmente empleados en el aula (Anexo I), según la opinión de los profesores, se recogen en la tabla II. En ella se observa: a) Prácticamente el $100 \%$ de los profesores en ejercicio $\mathrm{y}$ en formación considera que los problemas permiten desarrollar técnicas específicas de resolución (representar, calcular, manejar fórmulas y ecuaciones...).

b) Un alto porcentaje del grupo I $(84,8 \%)$ y aproximadamente la mitad de los otros colectivos (grupo II, 57,1\%; grupo III, 42,9\%) piensan que los alumnos suelen ana- 
Figura 2

Comparación entre las opiniones de los profesores sobre los objetivos que permiten desarrollar los problemas antes y después de analizar el problema tradicional.

\section{Grupo I}

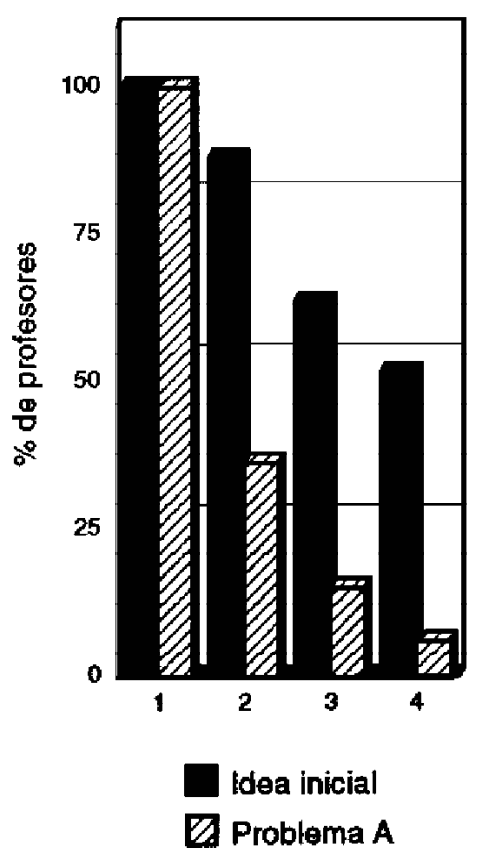

Grupo II

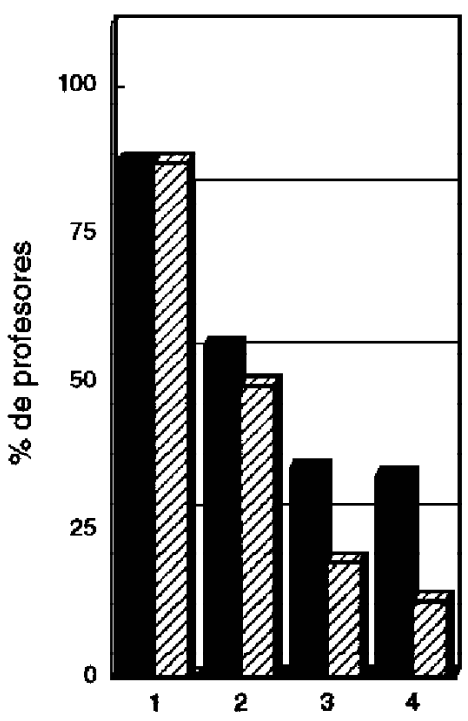

Idea inicial

Z Problema A
Grupo III

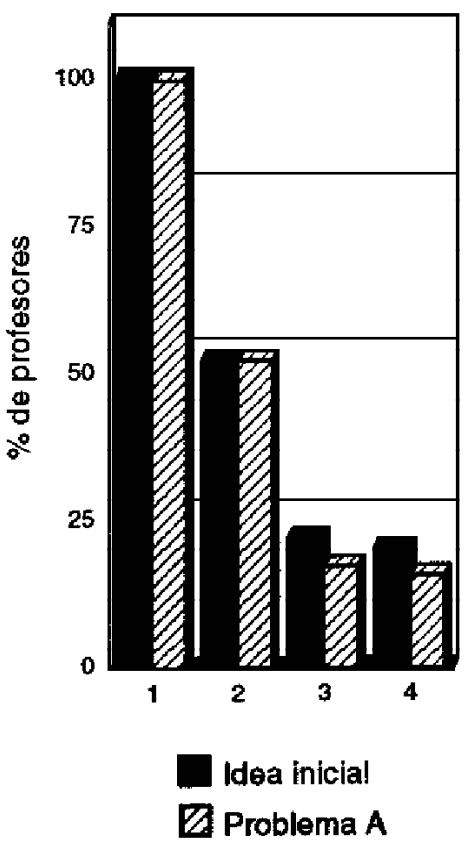

Tabla III

Valoración media, en rango de 0 a 10, otorgada por los profesores a posibles factores que limitan la capacidad educativa de los problemas habituales.

\begin{tabular}{|c|c|c|}
\hline POSIBLES CAUSAS & $\begin{array}{c}\text { CAP } \\
(n=44)\end{array}$ & $\begin{array}{l}\text { PROFESORES } \\
\quad(n=41)\end{array}$ \\
\hline No aplicación de la teoría & 8,3 & 7,9 \\
\hline Falta de trabajo personal & 5,3 & 8,4 \\
\hline Fallos en operaciones & 4,9 & 8,4 \\
\hline Complicación de los problemas & 2,3 & 1,7 \\
\hline No comprensión del enunciado & 7,3 & 8,6 \\
\hline Insuficiente preparación docente & 3,9 & 2,7 \\
\hline Dificultad para elaborar estrategias de resolución & 7,4 & 7,9 \\
\hline Falta de conocimiento o capacidad de los estudiantes & 7,3 & 7,6 \\
\hline
\end{tabular}


lizar los problemas y cuestiones planteadas utilizando los conocimientos teóricos disponibles. Se observaron diferencias significativas entre los alumnos de magisterio y los otros dos grupos de profesores $\left(\mathrm{X}^{2}=7,45\right.$ y 13,94 respectivamente).

c) Menos del $50 \%$ de los consultados de los tres colectivos reconoció que se desarrollaron procedimientos científicos esenciales en la resolución de problemas -propuesta de soluciones y diseño de estrategias, comprobación de hipótesis, análisis de resultados-. Se detectaron nuevamente diferencias significativas entre la opinión del grupo de magisterio y la posición, más crítica, de los alumnos del CAP y de los profesores en ejercicio (grupos II y III), respecto a que los estudiantes, ante un problema planteado, propongan posibles soluciones y diseñen estrategias de resolución $\left(\mathrm{X}^{2}=7,31\right.$ y 14,62).

d) La posibilidad de que los problemas desarrollen procedimientos relativos al ámbito de la comunicación (exponer por escrito el proceso seguido en la resolución de problemas, comunicar los resultados y discutirlos en gran grupo) fue reconocida asimismo por menos del $50 \%$ de los profesores de los distintos colectivos.

En la discusión en gran grupo que se realizó a continuación, los consultados justificaron sus opiniones con respecto a las limitaciones de los problemas para promover el aprendizaje. Tales limitaciones, expresadas mayoritariamente por los distintos colectivos y recogidas en el transcurso de la discusión, respondieron fundamentalmente a deficiencias del alumnado. A continuación se recogen frases textuales al respecto: no entienden el enunciado; si el problema no es similar a otro ya resuelto, no saben qué hacer para resolverlo; no saben aplicar a los problemas los conocimientos teóricos; no trabajan lo suficiente, les falta preparación o capacidad; fallos en operaciones. Debemos indicar que las referencias a las limitaciones de la propia enseñanza de resolución de problemas fueron esporádicas, sobre todo en el colectivo de profesores en ejercicio.

Como se ha indicado, algunos grupos de profesores, concretamente de los colectivos del CAP y de profesores en ejercicio, expresaron, a través de cuestionario cerrado (Anexo II), sus opiniones respecto a las causas que influyen en la limitada capacidad educativa de los problemas habituales. En la tabla III se recogen las citadas opiniones, apreciándose que los dos colectivos coinciden en otorgar una alta puntuación (valores superiores a 7 en un rango de 0 a 10) a factores directamente relacionados con la capacidad o habilidad de los estudiantes -falta de comprensión del enunciado, no saben aplicar a los problemas los conocimientos teóricos, no tienen claras las posibles vías o estrategias de resolución y no llegan a entender los problemas porque carecen de conocimientos o capacidades-. Por el contrario, ambos grupos han otorgado una puntuación baja a la insuficiente formación del profesor en la enseñanza de resolución de problemas $(3,9$ y 2,7$)$ y a la excesiva complicación de los problemas propuestos habitualmente $(2,3$ y 1,7$)$. Finalmente, debemos indicar que, si bien los profesores en ejercicio dieron también importancia a la falta de trabajo y esfuerzo personal y a los fallos en operaciones (la puntuación media obtenida fue de 8,4 en los dos casos), estos aspectos fueron menos valorados por los futuros profesores $(5,3$ y 4,9 respectivamente).

\section{Análisis y valoración de modelos de resolución tradicional y alternativo}

En la tabla IV se comparan la opiniones de los profesores respecto a los objetivos que promueven el problema tradicional A y el alternativo B.

El problema tradicional sirve, según los distintos colectivos, para aplicar la teoría, pero resulta insuficiente para alcanzar otros objetivos, especialmente para desarrollar capacidades y para interesar al alumno. El porcentaje de profesores que lo señaló no alcanzó el $20 \%$ en ningún grupo. Se debe indicar que las opiniones de los distintos grupos sobre el problema tradicional A fueron más negativas que las expresadas en el sondeo previo (Fig. 2), especialmente en el grupo de magisterio, en que los porcentajes descendieron significativamente para los objetivos relacionados con el aprendizaje y comprensión de conceptos $\left(\mathrm{X}^{2}=14,28\right)$, el desarrollo de capacidades $\left(\mathrm{X}^{2}=16,48\right)$ y el desarrollo de actitudes y hábitos $\left(X^{2}=14,49\right)$.

Los distintos grupos de docentes establecieron claras diferencias entre el problema A y el problema B. Así, para una gran proporción de los mismos (porcentajes superiores al $70 \%$ en todos los casos), el problema B, además de constituir un medio para aplicar la teoría, poseía mayores posibilidades educativas en cuanto al aprendizaje de conceptos y al desarrollo de capacidades, hábitos y actitudes necesarias para resolver problemas. Por otra parte, los consultados señalaron que el problema $\mathrm{B}$ resultaba potencialmente más motivador que el $\mathrm{A}$.

Las opiniones de los profesores respecto a los procedimientos que permiten desarrollar los problemas A y B se recogen en la tabla $\mathrm{V}$. Los distintos grupos consideraron mayoritariamente que el problema A permite ejercitar técnicas de resolución. Sin embargo éste no es idóneo, según la opinión de los tres colectivos, para promover otros procedimientos científicos. En este sentido destacamos que: a) su capacidad para que los alumnos propongan posibles soluciones y estrategias de resolución es reconocida únicamente por el $9,1 \%$ del grupo I, por el $10,4 \%$ del grupo II y por el $4,8 \%$ del grupo III; b) la posibilidad de que los estudiantes comprueben que se ha cumplido la hipótesis propuesta es admitida por menos del $15 \%$ de los distintos colectivos; c) su adecuación para promover el análisis de los resultados obtenidos fue sugerida por el 15,2\% del grupo I, el $15,6 \%$ del grupo II y el $34,9 \%$ del grupo III. Por otra parte, la exposición y discusión de ideas entre los estudiantes se ve favorecida en el problema A según el $26 \%$ del grupo II y el 38,1\% del grupo III, aunque sólo el $3,3 \%$ de los futuros maestros consideró tal aportación $\left(\mathrm{X}^{2}=6,46\right.$ y 12,065 respectivamente). En general, las opiniones de los profesores sobre la capacidad del problema tradicional A para desarrollar procedimientos 
Figura 3

Comparación entre las opiniones de los profesores sobre procedimientos que permiten desarrollar los problemas antes y después de analizar el problema tradicional A.
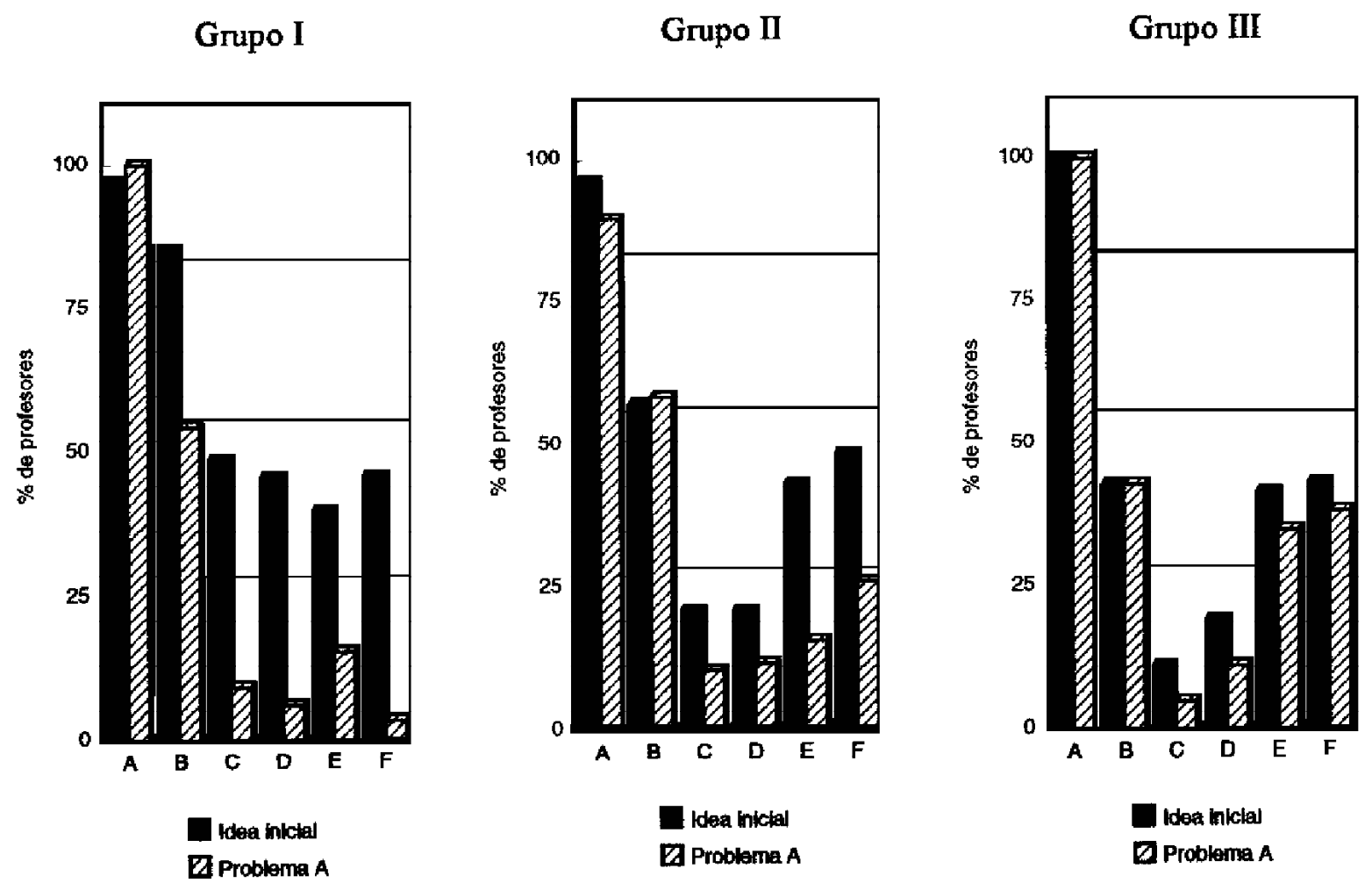

Procedimientos: A (aplicar técnicas); B (analizar el problema); C (proponer soluciones); D (comprobar hipótesis); E (analizar resultados); F (contrastar opiniones).

Tabla IV

Opinión de los profesores, expresada en porcentaje, respecto a los objetivos que se alcanzan en los problemas A y B.

\begin{tabular}{|c|c|c|c|c|c|c|}
\hline \multirow{3}{*}{ OBJETIVOS } & \multicolumn{3}{|c|}{ PROBLEMA A } & \multicolumn{3}{|c|}{ PROBLEMA B } \\
\hline & \multicolumn{3}{|c|}{ GRUPO } & \multicolumn{3}{|c|}{ GRUPO } \\
\hline & I & II & III & I & II & III \\
\hline Aplicar la teoría estudiada & 100 & 87, & 100 & 100 & 84,4 & 79,4 \\
\hline Favorecer la comprensión & 36,4 & 49,4 & 52,4 & 100 & 90,9 & 98,4 \\
\hline Desarrollar capacidades & 15,2 & 19,5 & 17,5 & 72,7 & 74,0 & 77,7 \\
\hline Interesar al alumno & 6,1 & 13,0 & 15,9 & 87,9 & 87,0 & 95,2 \\
\hline
\end{tabular}




\section{INVESTIGACIÓN DIDÁCTICA}

fueron también más negativas que las expresadas inicialmente respecto a los problemas escolares habituales (Fig. 3). Tales diferencias resultaron de nuevo más acusadas en el grupo I, siendo estadísticamente significativas para los procedimientos $\mathrm{C}$-propuesta de soluciones y diseño de estrategias-, D -comprobación de hipótesis- y F -contrastar opiniones- $\left(\mathrm{X}^{2}=10,64 ; 11,41\right.$ y 13,94).

En cuanto al problema innovador B, los distintos colectivos reconocieron que favorecía el desarrollo de procedimientos científicos en mucha mayor medida que el problema tradicional A. Cabe destacar aquí el alto porcentaje de sujetos de los tres colectivos que consideraron la capacidad del problema B para promover la propuesta de soluciones apriorísticas y el diseño de estrategias (93,9\% del grupo I; 93,5\% del grupo II; $100 \%$ del grupo III), así como la comprobación de las mismas (100\% del grupo I; 81,8\% del grupo II; 90,5\% del grupo III). El problema B tambien facilita el análisis de resultados y el intercambio de opiniones entre compañeros (más del $75 \%$ se manifestó en este sentido).

Debemos señalar que las consideraciones finales y la puesta en común ratificaron plenamente los resultados presentados. Los diferentes grupos de profesores valoraron positivamente el problema alternativo $\mathrm{B}$ y su modelo de resolución, criticando y cuestionando el problema tradicional A. Además, reconocieron la necesidad de que se produzca un cambio en el planteamiento y enseñanza de los problemas. La valoración del problema B se centró en una serie de razones que ejemplificamos a continuación utilizando frases textuales de profesores en ejercicio y en formación: hace pensar y entender el problema, se da más importancia al proceso de resolución, es más completo, favorece la resolución comprensiva del problema, es más motivador. Respecto a las críticas vertidas al problema $\mathrm{A}$, tambien recogemos frases textuales como: el problema A no lleva a la reflexión del alumno, con este tipo de enunciados se puede resolver el problema sin que sea necesario para ello comprenderlo o dominar la teoría, se debería ayudar al alumno a comprender el problema y a ser críticos con los resultados, deberían trabajarse otros contenidos procedimentales.

\section{DISCUSIÓN}

Consideramos que la actividad realizada con profesores en ejercicio y en formación ha resultado positiva. Los diferentes grupos se manifestaron en este sentido, resaltando el interés de este tipo de planteamiento centrado en la reflexión sobre la realidad y en el análisis de problemas tradicionales e innovadores. Ambos aspectos favorecen, por una parte, que se cuestione el pensamiento y la práctica docente habitual en el aula (Briscoe, 1991) y, por otra, que se utilice sistemáticamente el análisis crítico en la valoración de actividades.

En este trabajo pudimos observar que los profesores reconocen la capacidad educativa de los problemas, considerándose, en un principio, idóneos para aplicar la teoría a situaciones concretas, para favorecer el desarrollo de capacidades intelectuales de los estudiantes...

Tabla V

Opinión de los profesores, expresada en porcentaje, respecto a los procedimientos que permiten desarrollar los problemas A y B.

\begin{tabular}{|c|c|c|c|c|c|c|}
\hline \multirow{3}{*}{ PROCEDIMIENTOS } & \multicolumn{3}{|c|}{ PROBLEMA A } & \multicolumn{3}{|c|}{ PROBLEMA B } \\
\hline & \multicolumn{3}{|c|}{ GRUPO } & \multicolumn{3}{|c|}{ GRUPO } \\
\hline & $\mathbf{I}$ & II & III & I & II & III \\
\hline A - Ejercitar técnicas & 100 & 89,6 & 100 & 72,7 & 80,5 & 79,4 \\
\hline $\begin{array}{l}\text { B - Analizar el problema utilizando los conocimientos } \\
\text { teóricos disponibles }\end{array}$ & 54,5 & 58,4 & 42,9 & 100 & 100 & 96,8 \\
\hline $\begin{array}{l}\text { C - Proponer posibles soluciones y diseñar estrategias } \\
\text { de resolución }\end{array}$ & 9,1 & 10,4 & 4,8 & 93,9 & 93,5 & 100 \\
\hline $\begin{array}{l}\text { D - Comprobar que se ha cumplido la hipótesis } \\
\text { propuesta }\end{array}$ & 6,1 & 11,7 & 11,1 & 100 & 81,8 & 90,5 \\
\hline E - Analizar los resultados owbtenidos & 15,2 & 15,6 & 34,9 & 78,8 & 90,9 & 93,7 \\
\hline F - Contrastar opiniones con otros compañeros & 3,3 & 26,0 & 38,1 & 87,9 & 88,3 & 85,7 \\
\hline
\end{tabular}


Estas ideas de los profesores resultan coherentes con las aportaciones de la investigación sobre resolución de problemas, donde se destaca el potencial educativo de los mismos (Garrett, 1987; Perales 1993; Pozo et al., 1994,1995; Selvaratnman, 1983). Por otra parte, la valoración positiva de los problemas como actividades de aprendizaje está influida por la importancia real que tienen y han tenido los problemas en el aula. De hecho, los distintos modelos de enseñanza-aprendizaje de las ciencias, desde los más tradicionales hasta los más innovadores, contemplan los problemas como actividad obligada y específica, utilizándolos además como sistema de evaluación, lo que legitima y potencia, sin duda, la relevancia de los mismos.

Las altas expectativas manifestadas por los sujetos respecto a los problemas al inicio del trabajo, se matizaron cuando éstos realizaron el análisis guiado de «las posibilidades educativas reales de los problemas habituales de aula». Así, aunque los profesores de los distintos colectivos reconocieron mayoritariamente la utilidad de estas actividades escolares para aplicar los aspectos teóricos a situaciones concretas y para desarrollar técnicas específicas de resolución, tal reconocimiento no se extiende en la misma medida, entre los distintos grupos de profesores, a otro tipo de objetivos y procedimientos esenciales en la educación científica, siendo los profesores en ejercicio especialmente críticos en este sentido. Estas opiniones del profesorado en ejercicio, basadas en su propia experiencia docente, proporcionan una imagen pesimista de la realidad, corroborada por los pobres resultados que frecuentemente se obtienen con la realización de este tipo de actividades (Garrett, 1987; Genyea, 1983; Gil y Martínez Torregrosa, 1984; Gilbert, 1980; Mettes, 1980). Concretamente, con respecto al desarrollo de procedimientos, estos profesores se refirieron a que los alumnos no suelen realizar un análisis del problema y de los resultados obtenidos. En este sentido, destacaron que éstos generalmente se limitan a buscar relaciones algorítmicas entre los datos expresados en el enunciado y las fórmulas o definiciones estudiadas previamente. Con relación a otro tipo de procedimientos como la formulación de hipótesis y la comprobación de las hipótesis propuestas, los profesores en ejercicio fueron especialmente críticos. Esta postura puede responder al hecho de que los modelos de resolución de problemas presentados habitualmente en los textos escolares y en la propia aula, se caracterizan por una orientación centrada prioritariamente en la búsqueda del resultado correcto y en la adecuada utilización de los conceptos teóricos (Garrett et al., 1990), en detrimento de procedimientos tan relevantes en ciencias como la formulación de hipótesis, el diseño de estrategias de resolución, el análisis de resultados... En cualquier caso, la presencia de dichos procedimientos es todavía reducida en las distintas actividades de aula que en teoría resultan especialmente idóneas para ello como las actividades prácticas (García Barros et al., 1995; Tamir y García, 1992).

Respecto a los profesores en formación sería conveniente analizar las posibles causas que condujeron a los alumnos de magisterio a ser más optimistas respecto a las posibilidades reales de los problemas habituales que los alumnos del CAP. A nuestro juicio ha podido influir, entre otros aspectos, su propia formación teórica más amplia y específica en cuanto al ámbito psicopedagógico y a la didáctica de las ciencias. Tal formación motiva posiblemente que estos sujetos estén más familiarizados con los objetivos y contenidos específicos de la enseñanza de las ciencias, al menos en cuanto a su nomenclatura. También es posible que la insistencia puesta en su importancia educativa y la presentación de actividades específicas para favorecer su aprendizaje en el período de formación inicial promueva actitudes incluso «utópicas» respecto a las posibilidades educativas de las actividades de aula que, a menudo, son matizadas a lo largo del ejercicio profesional. Por el contrario, el hecho de que los alumnos del CAP se muestren más pesimistas con relación a qué objetivos y procedimientos desarrollan los problemas, podría responder a su propia experiencia, como alumnos de las facultades de ciencias. En ellas los planteamientos y los modelos de resolución de problemas empleados siguen respondiendo, en general, a los tipos más tradicionales, en los que los procedimientos científicos implicados son todavía insuficientes.

La discusión en gran grupo sirvió, como se pretendía, para poner en evidencia las limitaciones de los problemas como actividad orientada al desarrollo de conceptos y procedimientos científicos, pero además permitió discutir las posibles causas de dichas limitaciones. Si bien las opiniones de los profesores no fueron recogidas cuantitativamente en todos los grupos, éstas resultan básicamente coincidentes con las respuestas cuantitativas obtenidas en dos de ellos. Así, podemos afirmar que las causas sugeridas, tanto por los profesores en ejercicio como por los futuros docentes de secundaria, hacían referencia básicamente a las carencias y limitaciones de los estudiantes para enfrentarse a los problemas y resolverlos. Por el contrario, la influencia de la enseñanza fue apuntada solamente por algunos equipos de profesores, especialmente por los que se hallaban en período de formación inicial. Esta tendencia a centrar la problemática de la enseñanza-aprendizaje principalmente en los conocimientos, capacidades y habilidades del alumnado, minimizando la influencia del modelo de enseñanza, se encuentra bastante extendida entre el profesorado (Martínez Losada et al., 1993; Oñorbe y Sánchez, 1996). Tal tendencia ha sido detectada asimismo entre el alumnado que, además de reconocer sus limitaciones en la resolución de problemas, disculpa al profesor de su fracaso en este tipo de actividad (Oñorbe y Sánchez, 1996). El hecho de que los profesionales hayan dado más importancia que los profesores en formación a otros factores como la falta de trabajo y esfuerzo personal de los estudiantes o los fallos en operaciones puede deberse a que estos últimos tienen unas mayores expectativas con relación al alumnado, por encontrarse aún en período de formación inicial.

Lo indicado hasta el momento, unido al limitado análisis crítico de los docentes respecto a las deficiencias de la enseñanza de los problemas de ciencias, dificulta indudablemente la superación de las mismas. En nuestro planteamiento partimos, según se ha indicado, de las ideas de los docentes respecto a la enseñanza-aprendizaje 
de los problemas. Consideramos que tal reflexión es imprescindible pero claramente insuficiente si no se complementa con el análisis de modelos concretos de resolución de problemas. En esta ocasión utilizamos dos ejemplos, uno tradicional y otro innovador. Los profesores, como se recogió en los resultados, apreciaron las diferencias, destacando las mayores posibilidades educativas del segundo frente al primero y la importancia de su inclusión en el aula. Esta postura del profesorado podría responder a que el problema tradicional era el conocido «problema-tipo», mientras que el segundo problema, además de ser cualitativo, planteaba la realización del análisis del enunciado, la propuesta de hipótesis, la estrategia de resolución..., sugiriendo un sistema de resolución por investigación. Por otra parte, el análisis guiado del problema tradicional favoreció que los profesores profundizaran en sus limitaciones lo que, a nuestro juicio, contribuye a que se cuestionen opiniones iniciales «optimistas» respecto a este tipo de actividades, detectadas sobre todo en este trabajo entre los futuros maestros.

El hecho de que los profesores de los distintos colectivos hayan realizado una valoración positiva del modelo innovador de resolución de problemas es condición imprescindible para que éstos lo pongan en práctica en sus aulas y confirmen en ellas las potencialidades que encierra (Ramírez et al., 1994). Sin embargo, somos conscientes de que el cambio de orientación de los problemas requiere además un profundo cambio en su evaluación (Alonso et al., 1992; Dumas-Carré, 1987). Es tradicional que en ella se priorice el resultado, la corrección en los cálculos... frente a la estrategia de resolución, su justificación, el análisis de resultados... Estos últimos aspectos generalmente no se exigen en las pruebas de evaluación y, en el mejor de los casos, el docente los aprecia en el transcurso de la corrección. Por otra parte, debemos recordar que existen problemas de distintos tipos; las diferencias entre ellos radican, entre otras cosas, en los objetivos que persiguen y en los procedimientos que requiere su resolución. Si bien se ha critica-

\section{REFERENCIAS BIBLIOGRÁFICAS}

ALONSO, M., GIL, D. y MARTÍNEZ-TORREGROSA, J. (1992). Los exámenes de física en la enseñanza por transmisión y en la enseñanza por investigación. Enseñanza de las Ciencias, 10 (2), pp. 127-138.

BRISCOE, C. (1991). The dynamic interactions among beliefs. A case study of teacher change. Science Education, 72 (2), pp. 185-199.

CABALLER, M.J. y OÑORBE, A. (1997). Resolución de problemas y actividades de laboratorio, en La enseñanza y el aprendizaje de las ciencias de la naturaleza. Barcelona: ICE Universidad de Barcelona - Horsori. do la preponderancia exclusiva de los problemasejercicio en el aula, consideramos que su sustitución total por otro tipo de problemas más abiertos, que deban ser abordados de forma próxima a los problemas científicos, no sería deseable. Los distintos tipos de problemas deben tener su lugar en el proceso de enseñanza y su utilización ha de estar condicionada a las necesidades de los alumnos, al tipo de estudiantes, al tema concreto... En ocasiones será oportuno plantear ejercicios para que los alumnos apliquen o afiancen un determinado concepto, pero el profesor tiene que ser consciente de las limitaciones de este tipo de actividades y de la necesidad de plantear otros problemas que favorezcan la utilización y desarrollo de procedimientos científicos. Además, sería necesario que los docentes ampliaran el concepto de problema, habitualmente circunscrito a una actividad exclusivamente cognitiva, e incluir también problemas que impliquen algún tipo de actividad experimental en la línea propuesta por otros autores (Caballer y Oñorbe, 1997; Pozo et al., 1994; Watts, 1991). De acuerdo con todo lo anteriormente indicado, es imprescindible que el docente posea el suficiente nivel de formación que le permita analizar los distintos tipos de problemas y seleccionar aquéllos más idóneos para alcanzar los objetivos planteados. Es precisamente el análisis de modelos de resolución un aspecto fundamental del planteamiento presentado en este trabajo.

Finalmente, si bien, los docentes criticaron los problemas tradicionales y reconocieron la necesidad de utilizar propuestas innovadoras, sería conveniente preguntarse si este análisis de las actividades tradicionales y alternativas es suficiente para superar la inercia en la enseñanzaaprendizaje de problemas y reducir la preponderancia de los problemas operativos tradicionales en el aula. Consideramos que el cambio didáctico en la enseñanzaaprendizaje de problemas no es fácil (Gil y Pessoa, 1994); de acuerdo con ello, sería necesario dar un paso más en próximas investigaciones, con objeto de conocer en qué medida los profesores consolidan una nueva forma de abordar los problemas de aula.
CAILLOT, M. y DUMAS-CARRÉ, A. (1987). Un enseignement d'une méthodologie de résolution de problèmes en mathématiques et en physique. Rapports de Recherches, 12, pp. 199-244. París.

CAMACHO, M. y GOOD, R. (1989). Problem solving and chemical equilibrium: successful versus unsuccessful performance. Journal of Research in Science Teaching, 26 (3), pp. 251-272.

DUMAS CARRÉ, A. (1987). La resolution de problèmes en Physique an Licée. Thèse d'ètat sontenme à l'Université, Paris, 7. 
DUMAS CARRÉ, A. y LARCHER, C. (1987). The stepping stones of learning and evaluation. International Journal of Science Education, 9 (1), pp. 93-104.

FURIÓ, C. (1994). Tendencias actuales en la formación del profesorado de ciencias. Enseñanza de las Ciencias, 12 (2), pp. 188-199

GARCÍA BARROS, S., MARTÍNEZ LOSADA, C. y MONDELO, M. (1995). El trabajo práctico. Una intervención en la formación de profesores. Enseñanza de las Ciencias, 13(2), pp. 203-209.

GARRETT, R.M. (1986). Problem solving in Science Education. Studies in Science Education, 13, pp. 70-95.

GARRETT, R.M. (1987). Issues in science education: problemsolving, creativity and originality. International Journal of Science Education, 1, pp. 26-33.

GARRETT, R. M. (1988). Resolución de problemas y creatividad: implicaciones para el currículo de ciencias. Enseñanza de las Ciencias, 6(3), pp. 224-230.

GARRETT, R.M., SATTERLY, D., GIL, D. y MARTÍNEZTORREGROSA, J. (1990). Turning exercises into problems: an experimental study with teachers in training. International Journal of Science Education, 12 (1), pp. 1-12.

GENYEA, J. (1983). Improving student's problem-solving skills. A methodological approach for a preparatory chemistry course. Journal Chemical Education, 60, pp. 478-481.

GIL, D. y MARTÍNEZ-TORREGROSA, J. (1983). A model for problem solving in accordance with scientific methodology. European Journal of Science Education, 5 (4), pp. 447-455.

GIL, D. y MARTÍNEZ-TORREGROSA, J. (1984). Problem solving in Physics: a critical analysis. Research on Physics Education. París: Editions du CNRS.

GIL, D. y PESSOA, A. (1994). Enseñanza de las ciencias y la matemática. Madrid: Popular.

GILBERT, G. L. (1980). How do I get the ansewer. Journal of Chemical Education, 57, pp. 79-81.

HEWSON, P.W. y HEWSON, M.G. (1987). Science teachers' conceptions of teaching: implications for teachers education. International Journal of Science Education, 9(4), pp. 425-440.

KEMPA, R.F. (1986). Resolución de problemas de química y estructura cognitiva. Enseñanza de las Ciencias, 4(2), pp. 99-110.
KRAMERS-PALS, H., LAMBERCHTS, J. y WOLFF, P. J. (1983). The tansformation of quantitative problems to standard problems in general chemistry. European Journal of Science Education, 5(3), pp. 275-287.

LOPES, B. y COSTA, N. (1996). Modelo de enseñanzaaprendizaje centrado en la resolución de problemas: Fundamentación, presentación e implicaciones educativas. Enseñanza de las Ciencias, 14(1), pp. 45-61.

MARTÍNEZ LOSADA, C., GARCÍA BARROS, S. y MONDELO, M. (1993). Las ideas de los profesores de ciencias sobre la formación docente. Enseñanza de las Ciencias, 11(1), pp. 26-32.

METTES, C. T. C.W., PILOT, A., ROOSINK, J. H. y KRAMERS-PALS, H. (1980). Teaching and learning problem solving in Science. Part I: A general strategy. Journal of Chemical Education, 57, pp. 882-885.

OÑORBE, A. y SÁNCHEZ, J.M. (1996). Dificultades de la enseñanza-aprendizaje de los problemas de física y química. II. Opiniones del profesor. Enseñanza de las Ciencias, 14(3), pp. 251-260.

PERALES, F.J. (1993). La resolución de problemas: una revisión estructurada. Enseñanza de las Ciencias, 11(2), pp. 170178.

POLYA, G. (1965). Cómo planear y resolver problemas. Trillas: México.

POZO, J.I., PÉREZ, M.P., DOMÍNGUEZ, J., GÓMEZ, M.A. y POSTIGO, Y. (1994). Solución de problemas. Madrid: Santillana/Aula XXI.

POZO, J.I., POSTIGO, Y. y GONZÁLEZ CRESPO, M.A. (1995). Aprendizaje de estrategias para la solución de problemas de ciencias. Alambique. Didáctica de las Ciencias Experimentales, 5, pp. 16-26.

RAMÍREZ, J.L., GIL, D. y MARTÍNEZ-TORREGROSA, J. (1994). La resolución de problemas de física y química como investigación. Madrid: CIDE/MEC.

SELVARATNMAN, M. (1983). Student's mistakes in problem solving. Education in Chemistry, pp. 125-128.

TAMIR, P. y GARCÍA ROVIRA, M.P. (1992). Características de los ejercicios de prácticas de laboratorio incluidos en los libros de texto de ciencias utilizados en Cataluña.Enseñanza de las ciencias, 10(1), pp. 3-12.

WATTS, M. (1991).The science of problem-solving. A practical guide for science teachers. Londres: Cassell.

[Artículo recibido en julio de 1997 y aceptado en abril de 1998.] 


\section{ANEXO I}

Cuestiones orientadas a detectar ideas de los profesores en ejercicio y en formación respecto a los problemas de lápiz y papel.

Califica entre 0 y 10 (10 para las de mayor importancia) las posibles causas de la limitada capacidad educativa de los problemas habituales.

1. Objetivos que se suelen alcanzar con los problemas de lápiz y papel habituales

[ ] Aplicar la teoría que se ha explicado en clase o se ha estudiado previamente.

[ ] Favorecer el aprendizaje y comprensión de conceptos o de nuevas relaciones entre ellos.

[ ] Desarrollar la capacidad para abordar y resolver problemas reales y cotidianos.

[ ] Contribuir al desarrollo de hábitos y conductas deseables (hacerse preguntas, búsqueda de respuestas...) y, en definitiva, interesar al alumno por la resolución de problemas.

\section{Procedimientos que se desarrollan con los problemas de lápiz y papel habituales}

[ ] Ejercitar técnicas específicas: representar, manejar fórmulas y ecuaciones, calcular...

[ ] Analizar el problema o cuestión que se plantea, activando y utilizando los conocimientos teóricos disponibles.

[ ] Proponer y discutir posibles soluciones al problema planteado (hipótesis de trabajo) y diseñar estrategias para su resolución.

[ ] Comprobar que se ha cumplido la hipótesis propuesta y, por tanto, se ha resuelto el problema.

[ ] Analizar los resultados obtenidos de acuerdo con los datos contenidos en el enunciado del problema y confirmar que es lógico y coherente con la realidad.

[ ] Contrastar opiniones con otros compañeros (trabajo en grupo).

* [ ] Exponer por escrito el proceso seguido para la resolución.

* [ ] Comunicar los resultados obtenidos y discutirlos en gran grupo.

Nota: Esta misma guía se utilizó para recoger las opiniones de los sujetos respecto a los problemas tradicional A e innovador B. En estos dos casos no se incluyeron las preguntas marcadas con asterisco.

\section{ANEXO II}

Cuestionario para detectar las ideas de los profesores en ejercicio y en formación sobre las causas de la limitada capacidad educativa de los problemas habituales.

Califica entre 0 y 10 (10 para las de mayor importancia) las posibles causas de la limitada capacidad educativa de los problemas habituales.

[ ] Los alumnos no saben aplicar a los problemas los conocimientos teóricos.

[ ] Hay falta de trabajo y esfuerzo personal.

[ ] Fallos en operaciones, cálculos...

[ ] Los problemas que se plantean en el aula son demasiado complicados.

[ ] Falta de comprensión del enunciado del problema.

[ ] Insuficiente formación del profesor en la enseñanza de resolución de problemas.

[ ] El alumno no tiene claras las posibles vías o estrategias de resolución.

[ ] No llega a entender los problemas, le faltan conocimientos, capacidad... 


\section{ANEXO III}

Problemas propuestos para su análisis comparativo.

\section{Problemas tradicionales, tipo A}

¿Qué grupo sanguíneo tendrán los hijos de un hombre del grupo A, heterocigótico y una mujer del grupo B tambien heterocigótica? ¿Qué porcentaje de descendencia de cada gupo sanguíneo pueden tener?

Representamos el genotipo del hombre por $\mathrm{I}^{\mathrm{B}} \mathrm{i}$ y el de la mujer por $\mathrm{I}^{\mathrm{A}} \mathrm{i}$. A continuación realizamos los cruzamientos correspondientes. Combinando:

Progenitores $\quad \mathrm{I}^{\mathrm{A}} \mathrm{i} \quad \mathrm{x} \quad \mathrm{I}^{\mathrm{B}} \mathrm{i}$

Descencencia $\quad \mathrm{I}^{\mathrm{A}} \mathrm{I}^{\mathrm{B}} \quad \mathrm{I}^{\mathrm{A}} \mathrm{i} \quad \mathrm{I}^{\mathrm{B}} \mathrm{i} \quad$ ii

Como puede observarse, la proporción de genotipos de la descendencia es 1:1:1:1.

El 25\% de los hijos serán A, el $25 \% 0$ el $25 \%$ AB y el $25 \%$ B

Se lanza una piedra verticalmente hacia arriba con una velocidad inicial de $39,2 \mathrm{~m} / \mathrm{s}$. Halla: a) el tiempo que tarda en llegar al punto más alto; b) la altura máxima que alcanza la piedra.

a) Para calcular el tiempo que tarda la piedra en llegar al punto más alto podemos utilizar la ecuación: $\mathrm{v}=\mathrm{v}_{\mathrm{o}}+\mathrm{gt}$.

En el punto más alto, la velocidad final será,$v=0$. Además, debemos tomar el valor de $g$ con signo negativo porque la velocidad de subida y la gravedad tienen sentidos contrarios.

Sustituyendo, obtenemos: $0=39,2 \mathrm{~m} / \mathrm{s}+\left(-9,8 \mathrm{~m} / \mathrm{s}^{2}\right) \cdot \mathrm{t}$

Despejando: $\mathrm{t}=\frac{39,2 \mathrm{~m} / \mathrm{s}}{9,8 \mathrm{~m} / \mathrm{s}^{2}}=4 \mathrm{~s}$

b) La altura máxima a la que llega la piedra puede calcularse ahora a partir de la ecuación del espacio: $\mathrm{s}=\mathrm{v}_{\mathrm{o}} \mathrm{t}+1 / 2 \mathrm{gt}^{2}$

Recordemos que $g$ es negativa.

$$
\mathrm{s}=39,2 \mathrm{~m} / \mathrm{s} \cdot 4 \mathrm{~s}+1 / 2(-9,8) \mathrm{m} / \mathrm{s}^{2} \cdot 4^{2} \mathrm{~s}^{2}=78,4 \mathrm{~m}
$$

El espacio recorrido es de 78,4 m.

\section{Problemas innovadores, tipo B}

Si se lanza un objeto verticalmente hacia arriba, ¿qué altura máxima alcanzará?

a) Realizad un análisis de la situación planteada y del interés de su estudio.

Nota: Debe insistirse en la necesidad de una descripción cualitativa previa a cualquier planteamiento cuantitativo con el fin de favorecer la comprensión de la misma. Ésta puede acompañarse de una visualización mediante la construcción de un esquema gráfico e incluso la puesta en práctica del movimiento en cuestión.También será preciso acotar el problema, suponiendo despreciable el rozamiento del aire.

b) ¿De qué factores depende que el cuerpo alcance una mayor o menor altura? Intenta establecer las oportunas relaciones.

Nota: Esta actividad permite que los alumnos manifiesten sus ideas. Pueden referirse a aspectos como «cuanto mayor es la velocidad con la que se lanza el cuerpo, más arriba sube», «si no hubiese gravedad, el cuerpo no frenaría, por tanto, cabe pensar que la altura alcanzada dependerá de $g$ ». También es posible que incluyan otros factores, como, por ejemplo, la masa del cuerpo. En ese caso el profesor deberá favorecer el análisis crítico de esas afirmaciones (propondrá el intercambio de opiniones, planteará contraejemplos, sugerirá soluciones...).

c) Elaborad una estrategia de resolución de acuerdo con la hipótesis propuesta.

Nota: Los alumnos pueden optar por una estrategia del tipo: «Considerando que se trata de un movimiento uniformemente variado y conociendo las ecuaciones de la posición y de la velocidad del objeto a lo largo de la trayectoria en función del tiempo, se podría calcular el instante en que llega a la altura máxima (a partir de la ecuación de la velocidad) y sustituir dicho valor en la ecuación de la posición.»

d) Resolved ahora el problema planteado. Procurad explicar cómo concretáis y lleváis a cabo el plan de resolución elaborado.

Nota: Deberá resaltarse la necesidad de que la resolución se lleve a cabo verbalizando al máximo y evitando la simple utilización de las ecuaciones correspondientes. 


\section{INVESTIGACIÓN DIDÁCTICA}

e) Analizad los resultados obtenidos: ¿están de acuerdo con la hipótesis formulada?, ¿se obtiene el mismo resultado utilizando una estrategia de resolución diferente?, ¿el resultado es coherente y lógico con la realidad?..

Nota: Es importante realizar aquí una puesta en común en la que se recojan y contrasten las aportaciones de los distintos grupos.

f) Como actividad de aplicación, ¿podéis calcular ahora la altura máxima que alcanzará el cuerpo si su velocidad inicial era de 20 m/s?

Dos personas separadas desde la niñez sospechaban que podrían ser hermanos. ¿Podrían realmente serlo teniendo en cuenta que sus grupos sanguíneos son el AB y el O respectivamente?

a) Realizad un análisis de la situación y del interés de su estudio.

Nota: Debe insistirse en la necesidad de una descripción cualitativa previa a cualquier planteamiento cuantitativo con el fin de favorecer la comprensión de la misma. También debe resaltarse que la comprensión del enunciado del problema requiere que los alumnos reconozcan lo que significa pertenecer a determinados grupos sanguíneos, que éstos son caracteres heredables codificados por tres alelos de un gen (Ia, Ib, Io) y que cada uno de los alelos proviene de un progenitor.

b) Proponed posibles hipótesis al problema planteado.

Nota: Esta actividad permite que los alumnos manifiesten sus ideas. Pueden referirse a aspectos como «dado que Ia e Ib son dominantes frente a Io, que es recesivo, y que Ia e Ib son codominantes, estas personas podrían ser hermanos. Sin embargo, los demás posibles hermanos podrían poseer cualquier otro grupo sanguíneo». También es posible que se realicen afirmaciones inadecuadas. En ese caso, el profesor deberá favorecer el análisis crítico de las mismas (propondrá el intercambio de opiniones, planteará contraejemplos, sugerirá soluciones...).

c) Elaborad una estrategia de resolución de acuerdo con la hipótesis propuesta.

Nota: Los alumnos pueden optar por una estrategia del tipo: «Habrá que descubrir qué alelos tendrán que poseer los padres de cada uno de los presuntos hermanos y averiguar si la misma pareja podría ser padres de ambos. Posteriormente, estudiando los posibles cruzamientos de los padres, se podría averiguar si todos su hijos han de responder a los grupos sanguíneos de los presuntos hermanos o si, por el contrario, pueden pertenecer a cualquier otro grupo.»

d) Resolved ahora el problema planteado. Procurad explicar cómo concretáis y lleváis a cabo el plan de resolución elaborado.

Nota: Deberá resaltarse la necesidad de que la resolución se lleve a cabo verbalizando al máximo y evitando la simple utilización de las clásicas representaciones.

e) Analizad los resultados obtenidos: ¿están de acuerdo con la hipótesis formulada?, ¿el resultado es coherente y lógico con la realidad?...

Nota: Es importante realizar aquí una puesta en común en la que se recojan y contrasten las aportaciones de los distintos grupos.

f) Como actividad de aplicación podéis calcular ahora el porcentaje de descendencia de cada grupo sanguíneo que pueden tener esos padres. 